\title{
Investigating the Spatiotemporal Imbalance of Accessibility to Demand Responsive Transit (DRT) Service for People with Disabilities: Explanatory Case Study in South Korea
}

\author{
Jong-Hun Son $\mathbb{D}^{1},{ }^{1}$ Do-Gyeong Kim $\mathbb{D}^{2},{ }^{2}$ Eunkyeong Lee $\mathbb{D}^{3},{ }^{3}$ and Hosik Choi $\mathbb{C}^{3}$ \\ ${ }^{1}$ Department of Transportation Engineering, University of Seoul, Seoul 02504, Republic of Korea \\ ${ }^{2}$ Department of Transportation Engineering \& Graduate School, Department of Urban Big Data Convergence, \\ University of Seoul, Seoul 02504, Republic of Korea \\ ${ }^{3}$ Department of Urban Big Data Convergence, University of Seoul, Seoul 02504, Republic of Korea \\ Correspondence should be addressed to Do-Gyeong Kim; dokkang@uos.ac.kr
}

Received 1 September 2021; Revised 6 December 2021; Accepted 21 December 2021; Published 6 January 2022

Academic Editor: Chansung Kim

Copyright ( $\odot 2022$ Jong-Hun Son et al. This is an open access article distributed under the Creative Commons Attribution License, which permits unrestricted use, distribution, and reproduction in any medium, provided the original work is properly cited.

\begin{abstract}
This study analyzed the operation-related historical data of the call taxi service for disabled people in Seoul, South Korea. The study investigated how unevenly distributed the accessibility of disabled people to transportation is in terms of time and space. In addition, the reasons that cause imbalanced accessibility were investigated in areas with good and poor accessibility. Accessibility was defined as how quickly call taxi services for the disabled are available at specific times and locations. For the analysis, the log data for tracking the status of taxis in time and space were processed to calculate their availability, an index that reflects the dwelling time and the number of taxis available at a specific time and in a specific area. This index was divided into time and space and used as a surrogate measure to assess accessibility. The results showed that there were spatial and temporal accessibility imbalances in demand responsive transit (DRT) service. The insufficient supply during the night resulting from the current DRT operating schedule has reduced the accessibility of call taxis for the disabled, and the concentration of drivers' breaks also affected the accessibility of service during the daytime. This suggests the need for (1) an increase in supply and (2) evenly distributed breaks for the drivers. In terms of space, the outer areas of Seoul generally were found to be more accessible than the central areas. In addition, areas near depots that serve as hubs and resting places for taxi drivers, areas with excellent medical infrastructures for people with disabilities, and areas with good traffic environments tended to have good accessibility; this suggests the need to reallocate garages and improve the traffic environments to improve accessibility.
\end{abstract}

\section{Introduction}

All people should be guaranteed the right to free and safe mobility in order to conduct social and economic activities as members of society. This right is called the right to mobility, and it is one of the fundamental human rights that people deserve and exercise as members of society. The Universal Declaration of Human Rights also states the following, i.e., "Everyone has the right to freedom of movement and residence within the borders of each state" [1].

However, groups of people who have reduced mobility and use the road, such as physically disabled and elderly people, have significant limitations in participating in social activities because, compared to normal adults, they do not have the ability to move safely and freely [2]. Therefore, many countries provide special transportation services, such as demand responsive transport (DRT) to ensure equal mobility rights for people who must use the roads but have limited mobility. Representative DRT services for the disabled include access-a-ride (AAR) in New York, U.S., Telebus in Berlin, Germany, Optibus in Lyon, France, wheeltrans in Toronto, Canada, and handy-DART in Vancouver, Canada.

South Korea also defines the right to mobility (the term "Right to Transportation" is defined as "in order to be 
guaranteed the right to pursue the dignity, value, and happiness as a human being, mobility disadvantaged persons shall have the right to use safely, conveniently, and without discrimination, all means of transportation, passenger facilities, and roads used by persons, other than the mobility disadvantaged persons" in Article 3, Act on Promotion of the Transportation Convenience of Mobility Disadvantaged Persons) in the Act on Promotion of the Transportation Convenience of Mobility Disadvantaged Persons to guarantee the mobility rights of all people who use the road, such as the physically disabled and the elderly. This act was enacted as a part of the efforts to create a safe and convenient transportation environment for mobility-disadvantaged people. Among these efforts, DRT services have been provided as a special means of transportation to ensure the mobility rights of disabled people.

Free access to transportation should be guaranteed for the disabled so they can fully enjoy their mobility rights without discrimination. In other words, accessibility to transportation is an essential element to ensure equal mobility rights for the disabled. Therefore, many studies have been conducted to improve the accessibility for people with disabilities. A study by Casas (2007) dealt with the accessibility of the disabled from a socioeconomic point of view [3], whereas Bakker and Hal attempted to identify the travel characteristics of the disabled that affect accessibility [4]. Several studies were conducted to deliver policy implications to decision-makers by applying various analysis techniques $[5,6]$.

However, most of the previous studies on the accessibility of transportation for disabled people have focused mainly on facilities that create barrier-free or obstacle-free physical environments. They insisted that efforts should be made to improve the physical environments to enhance the accessibility of the disabled by managing walking environments such as sidewalk pavement [7] and by providing convenient access to large-scale public facilities such as hospitals [8], terminal and transfer centers [9-11].

Social interest in easy access to transportation means and facilities is necessary to guarantee complete mobility rights for the disabled, but there is a lack of research on disabled people's accessibility to the various forms of transportation. Among the few studies that have been conducted, some have included developing a business model of a mobile service transportation platform for the disabled [12], and some have included research on the barriers to providing DRT services for disabled people [13].

Accessibility to transportation can be interpreted in many ways, but the definition in this study is how easy and convenient it is for disabled people to use transportation services. Access to transportation is considered very good if people with disabilities can easily use transportation services at any time, and it is very important to guarantee the right to mobility without discrimination regardless of where people live.

However, in reality, accessibility to transportation is not guaranteed equally, so imbalances occur in that accessibility is distributed extensively in specific areas and time periods. This might be due to the characteristics of DRT services that do not have fixed routes or timetables but operate depending on the requests made by passengers.

The aim of this study was to investigate how unevenly distributed the accessibility to transportation means for disabled people is over time and space by analyzing the operation-related historical data of call taxi services for the disabled in Seoul, South Korea. In other words, this study tried to identify whether the DRT services are equally provided at any time to the disabled people who are willing to use them, regardless of where they live. For the analysis, an index called "availability," which is defined as a surrogate measure for assessing the accessibility to transportation services, was used in this study because this index indicates how easily and quickly DRT services for the disabled are available at a specific time and area. The results of this analysis might be expected to contribute to the establishment of policies related to the mobility of disabled people.

\section{Seoul Demand Responsive Transit (DRT) Service for the Disabled: Overview}

The Seoul metropolitan government started DRT services with 100 vehicles in 2003 to guarantee and promote the mobility rights of persons with disabilities. As of August 2021, Seoul's DRT services are operating a total of 685 vehicles. As specified in article 16 of the act on the promotion of the transportation convenience of mobility disadvantaged persons, these vehicles are special means of transportation, and, unlike other types of DRT services, most of them are equipped with slopes or lifts for people in wheelchairs because these vehicles are available only for people with severe disabilities.

DRT service is available 24 hours a day, 365 days a year. Those who wish to use the DRT service are required to register as a member. After registering, they can request service by calling the call center at any time. In principle, this service is available only within Seoul, but it can be extended to the metropolitan area near Seoul when transporting people to rehabilitation facilities for medical treatment. The basic or minimum fare is about 1.25 U.S. dollars for distances up to $5 \mathrm{~km}$, and there are additional charges for distances that exceed $5 \mathrm{~km}$.

The supply of vehicles is adjusted according to weekdays, weekends, and the time of day considering the users' needs. As shown in Figure 1 below, vehicles are allocated according to the time of day, and the number of available vehicles is reduced by $50 \%$ on weekends. However, the number of vehicles available at night time is the same on weekdays and weekends (Figure 1).

As of August 2021, there are 41 depots to park or store call taxis for the disabled in Seoul, and each depot has an average of about 15 vehicles. The call taxi drivers drive the vehicles and return to the depot after work. Figure 2 shows the spatial distribution of DRT depots across the city of Seoul.

\section{Data Description}

3.1. DRT Service Data. In this study, we used the $2019 \log$ data of call taxis for the disabled, which was before the 


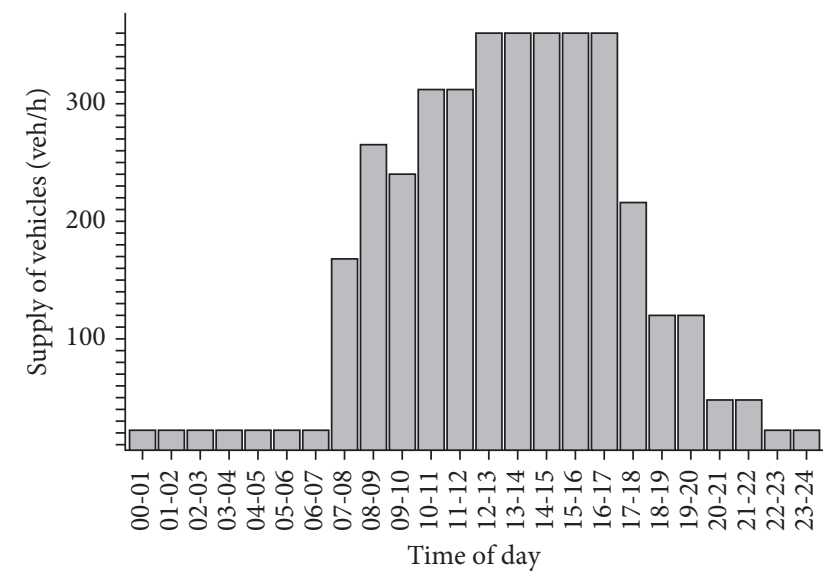

FIGURE 1: Number of DRT vehicles operated by time of day (August 2021 weekdays).

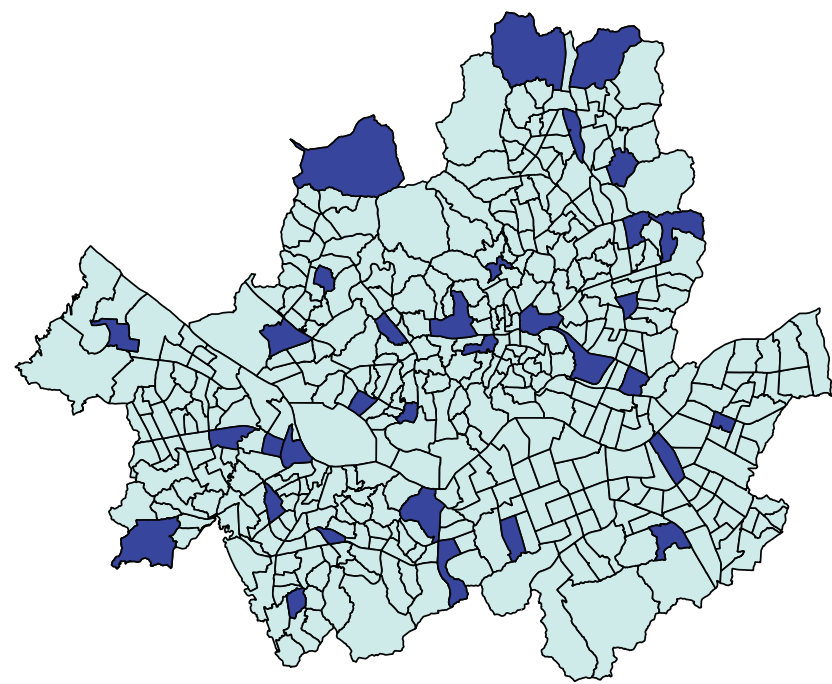

FIGURE 2: Spatial distribution of call taxi depots for the disabled in Seoul.

changes in traffic volumes and behaviors caused by the COVID-19 pandemic. The log data were divided into three main categories, i.e., (1) member information data containing user information, (2) operation data recording the origin and destination of trips, boarding time, and dispatch time, and (3) service data showing the status and location of each vehicle.

Of the three types of data, the service data of each vehicle were used in this study because it tracks the status and location of each vehicle minute-by-minute, resulting in about 250,000 cases per day and 90 million cases per year. To be more specific, the service data were collected every minute, and they included the status of the vehicle, the times when the data were collected, vehicle identification number, and the latitude and longitude location of the vehicle. The status of the vehicle is divided and coded into 15 types or categories indicating, for example, whether or not passengers are in the vehicle, and whether the driver is on break. The categories include "dispatched," "passenger on board," "waiting for boarding," "accident occurred," "driver on break," and "vacant vehicle."
3.2. Pre-Processing of the Data. In principle, call taxis for the disabled only operate within Seoul, but, as mentioned above, sometimes they extend their service to adjacent metropolitan areas at the request of users. However, this study limited the spatial scope to Seoul and only used the service data generated within Seoul. Also, among the 15 coded vehicle status types, the log data for "accident occurred," "dispatch refused," and "boarding failure" were excluded from the analysis because they indicate relatively special states or have low direct relevance with the provision of DRT service.

3.3. Data for Final Analysis. After excluding $4,169,835$ bits of data from the pre-processing process , about 85,492,131 bits of vehicle service data were used for the final analysis. These data were recorded once per minute by the DRT in service, so it indicates the service time. A total of 134,477 call taxis for the disabled (about 637 per vehicle) recorded these data, which means that each vehicle was in service for about 10.6 hours.

During the total service hours, the percentage of "inservice," where the driver is reserved or already has been assigned to another customer, was $79.3 \%$, and the ratio of logs indicating "driver break" was about $6.6 \%$. The "vacant vehicle" status, which means that the car is available to users, accounted for $14.1 \%$ of the total service time.

Based on the abovementioned information, the average "vacant" status per vehicle was about 1.5 hours/vehicle per day. The average "in-service" status per day and the average "driver break" status per day was 8.2 hours/vehicle and 0.7 hours/vehicle, respectively.

\section{Analysis and Assessment of Accessibility}

4.1. Definition of Accessibility Index. Bhat et al. suggested various types of models to quantitatively (numerically) express the accessibility based on the results of many related studies [14]. Based on the models, prior studies have defined accessibility as the number of major facilities $[15,16]$ or jobs [17], travel distance [18], travel time [19-21], and the number of people who can be transported [22].

In this study, accessibility was defined as how easily and conveniently a person with reduced mobility can use transportation services (means) necessary to move. To be more specific, this definition means accessibility in terms of time, indicating how quickly people with disabilities can use the service without waiting for a long period. That is if there are many vehicles available at time $j$ in area $i$, the accessibility to transportation services in that area and at that time is considered to be very good. Also, even if only a few vehicles are available, the accessibility to transportation services is considered good if vacant vehicles are waiting for a long time in the same area.

In this respect, this study intends to explain the accessibility to DRT service by using an alternative variable, i.e., availability. As shown in (1), the availability at time $j$ in the area $i$ can be expressed as a combination of the number of vacant vehicles and the dwell time in vacant status per vehicle. The dwell time can be defined as the time the driver 
waits for a call to be dispatched after one operation is completed.

$$
\begin{aligned}
\text { availability }_{i j}= & \left(\text { dwell time in empty per vehicle }_{i j},\right. \\
& \text { number of empty vehicles } \\
i j & ),
\end{aligned}
$$

where $i$ refers to the administrative district, and $j$ is the time of the day. That is, the availability at time $j$ in area $i$ is explained by two factors, i.e., (1) how many vehicles are vacant and (2) how long each vehicle stays vacant in the corresponding time and space.

4.2. Calculation of Availability Index. Based on (1), the availability, which is used as a surrogate measure of accessibility to transportation, was calculated for 431 administrative districts in Seoul by time of day to analyze accessibility, and a total of 10,344 availabilities were obtained for each district and timeof day. Based on the location and time of the vehicle that is generated every minute and recorded in the vehicle service data log, the log records of the vehicles were divided by time and administrative district, and only the codes for "vacant vehicle" were summed to calculate the average time when each vehicle was vacant. Then, based on the vehicle identification numbers in the service data log, the number of vacant vehicles for each time and space was derived by considering the logs left by the same vehicle as one. The final number of vacant vehicles and the average time each vehicle was vacant were derived for each temporal and spatial category. After excluding cases in which the number of available vehicles was 0 or there were missing values with no data, 10,253 availabilities were used for the analysis out of 10,344 temporal and spatial categories.

After setting the time each vehicle was vacant as the $x$ axis and the number of vacant vehicles as the $y$-axis and dividing each axis by 10 percentiles into 10 classes, 10,253 availabilities were assigned to each class, and the frequency of availability was calculated by time and administrative district for each class, as shown in Figure 3. Four quadrants were created by dividing the areas based on the $50^{\text {th }}$ percentile of the $x$ and $y$ axes, and the meaning of each quadrant was as follows:

Quadrant I: Areas with many vacant vehicles, but each vehicle has a short dwelling time.

Quadrant II: Areas with many vacant vehicles and the dwelling time per vehicle is long.

Quadrant III: Areas with fewer vacant vehicles and the dwelling time per vehicle is short.

Quadrant IV: Areas with fewer vacant vehicles, but each vehicle has a long dwelling time.

By considering the meaning of each quadrant, quadrant II can be interpreted as areas with good accessibility because vehicle availability is very good, whereas quadrant III represents areas with poor accessibility due to the low availability of vehicles.

The box plot below (Figure 4) is based on dwell time (Figure 4(a)) and the number of vacant vehicles (Figure 4(b))

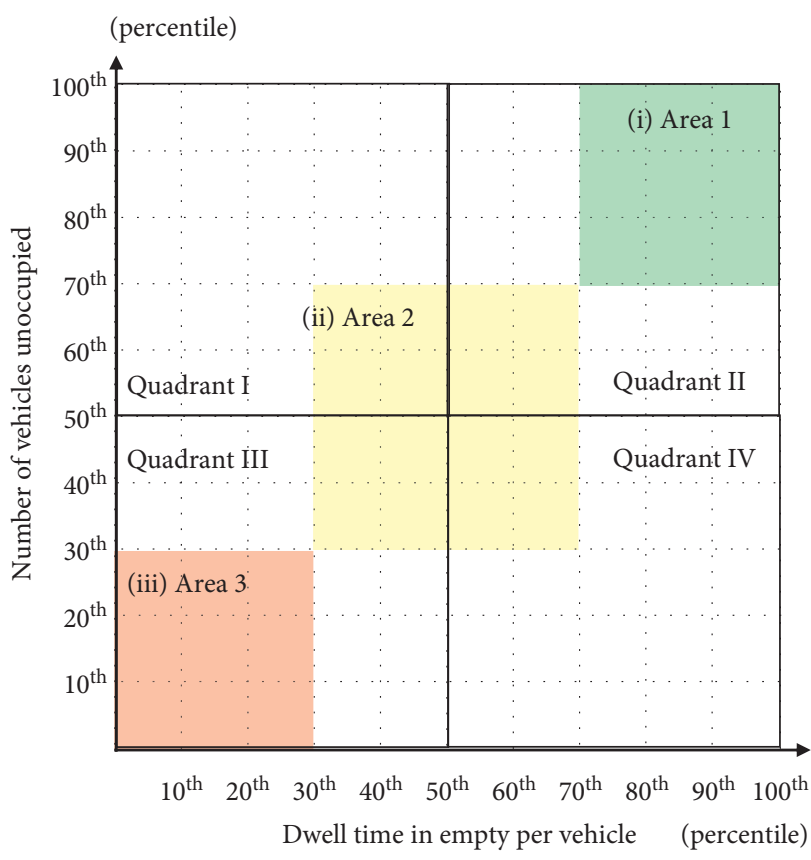

FIgURE 3: Two-dimensional availability.

to understand the distribution of data for each quadrant. The distribution of dwell time in quadrant I and quadrant III with the same level of dwell time was similar. In quadrant IV, the median value was relatively similar to that of quadrant II, but the mean value was large and biased to the right. This is because quadrant IV has more values with relatively high dwell times. In terms of the number of vacant vehicles, even though quadrants I and II share the same range, the difference in distribution showed that the mean of quadrant II was larger than the mean of quadrant I. This also was because quadrant II had a large distribution of availability with many vacant vehicles.

4.3. Analyzing the Spatiotemporal Characteristics of Accessibility. In this study, we investigated whether disabled people have equal access to transportation services in terms of time and space, and we analyzed the reasons that cause imbalances. Figure 3 shows that the accessibility to the corresponding transportation service is not equal in time or space. Therefore, this section uses the data that belong to quadrant II with good accessibility and quadrant III with poor accessibility to analyze the factors causing such differences in terms of time and space. In addition, the temporal characteristics of accessibility for quadrant I and IV are briefly explained.

For a clearer analysis, the areas in which the values on both axes are above the $70^{\text {th }}$ percentile in quadrant II were defined as the good group (green area in Figure 3, (i) area (1)), and the areas where the values on both axes are less than or equal to the $30^{\text {th }}$ percentile in quadrant III as the bad group (red region in Figure 3), (iii) area (3)). The data from both groups were used for analysis. The values around the $50^{\text {th }}$ percentile (or median) (yellow region in Figure 3, (ii) area (2)) were excluded from the analysis because it was 


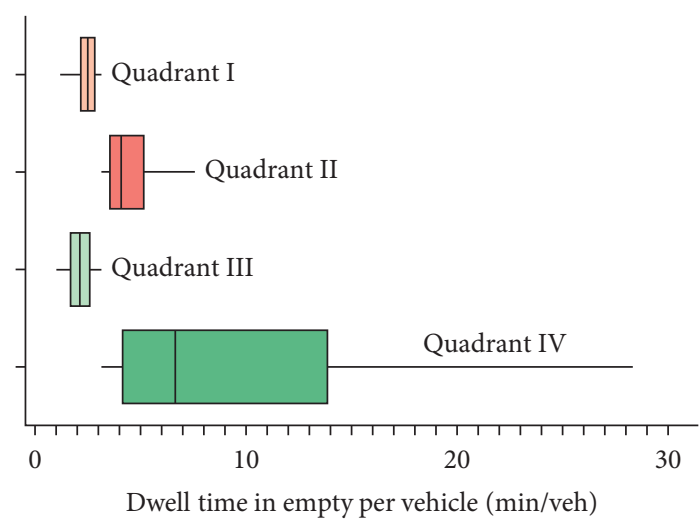

(a)

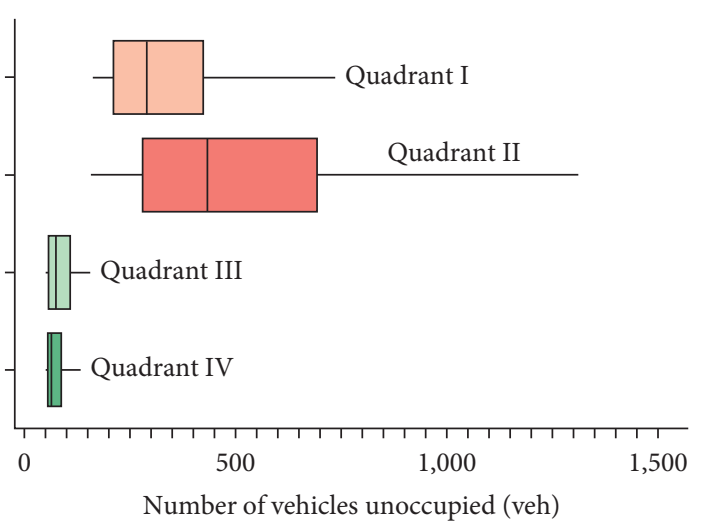

(b)

FIGURE 4: Box plot of availability by each quadrant: (a) based on dwell time; (b) based on the number of unoccupied vehicles.

difficult to clearly explain whether the accessibility was good or bad.

4.4. Analysis of the Temporal Characteristics. As described previously , the good group refers to a time and administrative district where accessibility or the availability of vehicles was very high, and the bad group means a time and administrative district where the availability of vehicles was very low. To examine the temporal characteristics between the two groups, the availability indicators associated with the good and bad groups were integrated by time to compare the share of each group according to time, as shown in Figure 5. In the figure, red indicates the bad group, and green indicates the good group.

The results of the analysis show that most of the availability during the late-night period, i.e., from 10:00 PM to 5:00 AM, belongs to the bad group, which means that the accessibility during the late-night time is very poor. However, most of the availability during the daytime belongs to the good group, indicating very good accessibility.

The reason for this might be the fact that the current supply of DRT service in Seoul is not equal at different times. As shown in Figure 1, the distribution of vehicles in service by time of day is low during the late-night and early morning hours, but the distribution is high during the daytime. That is, the higher the number of vehicles in service, the better the accessibility to the DRT service. Considering the availability of vehicles compared to the travel demand for each time period, the accessibility to the DRT service during the nighttime might be expected to be much better, but opposite results were obtained due to the fact that there were many regions where the number of vehicles operated during the nighttime is zero. These results suggest the need to increase the supply to improve accessibility.

Another temporal characteristic is the poor availability in some daytime hours (12:00 to 2:00 PM). As shown in Figure 1, more vehicles are allocated during these hours than in other periods, but the availability is poor because most drivers take breaks in the same period.

Figure 6 represents the total amount of time spent on "driver breaks" (bar chart) and break time per each vehicle (solid line) by time of day. The average break time per vehicle

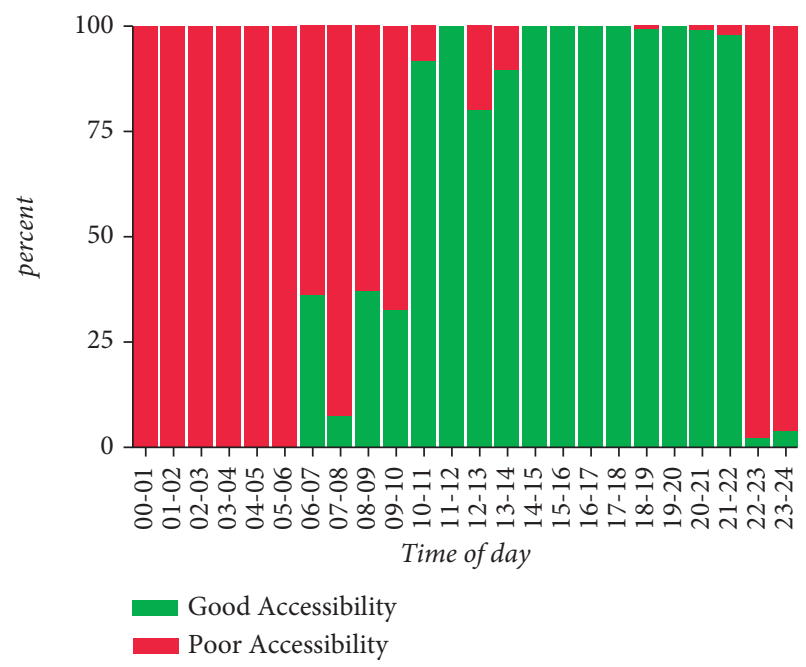

Figure 5: Ratio of good and bad groups by time of day.

was found to be relatively high from midnight to dawn (12: 00 to 6:00 AM) and slightly increased during some daytime hours (12:00 to 2:00 PM). The distribution of break time per vehicle by time of day was found to have a very similar pattern with the ratio of good and bad accessibility by time of day as shown in Figure 5, indicating that the driver's break time might contribute to the poor accessibility in the corresponding time period to some extent. To solve this problem, it is necessary to prevent the concentration of drivers' breaks during certain hours and distribute them evenly according to time.

The availability characteristics of regions and time periods classified into quadrant I can be defined as a state in which a large number of vehicles with a relatively short dwell time in empty per vehicle exist. On the contrary, few vehicle with a long dwell time in empty per vehicle is a typical characteristic of quadrant IV. These characteristics might affect the temporal distribution of availabilities belonging to quadrant I and IV showing the opposite trend. As shown in Figure 7, the availabilities of quadrant I are temporally distributed from early morning to midnight, whereas quadrant IV is dominated by time periods from midnight to dawn. 


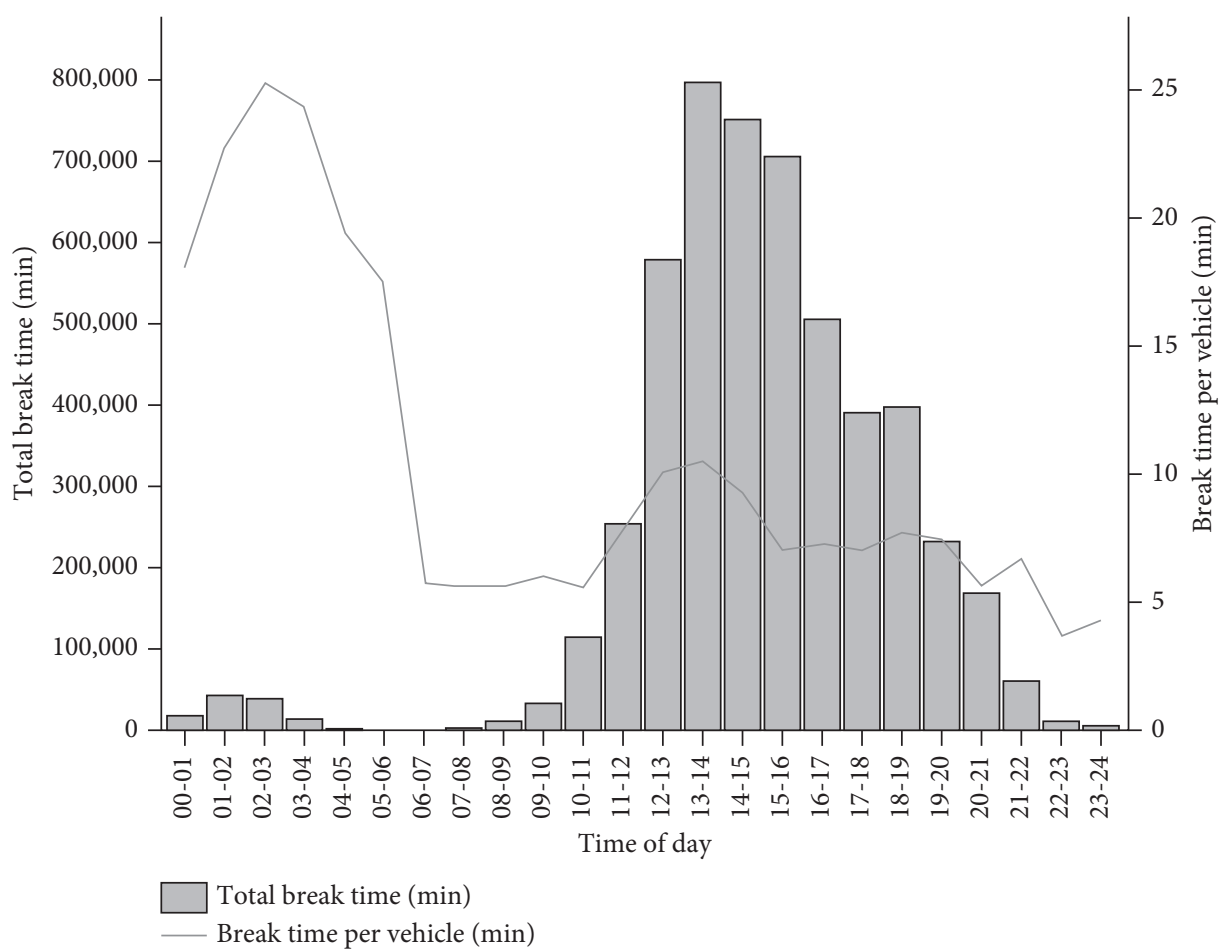

FIGURE 6: Distribution of drivers' breaks by time of day.

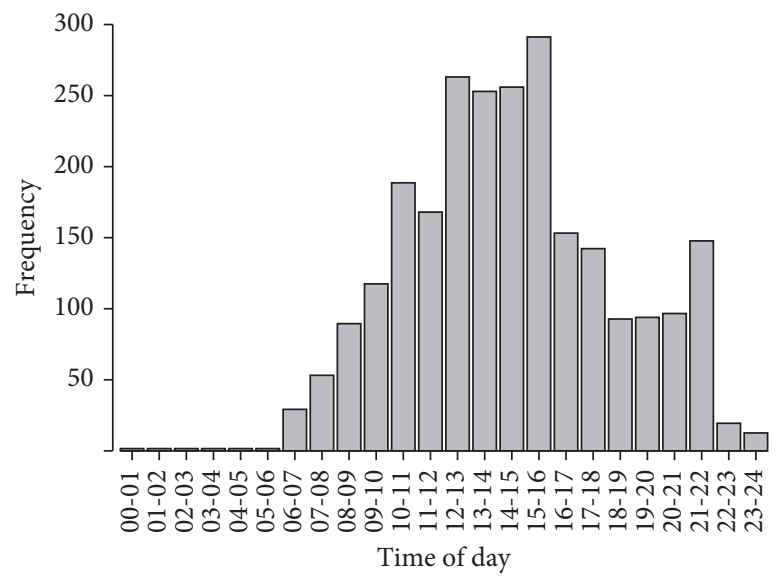

(a)

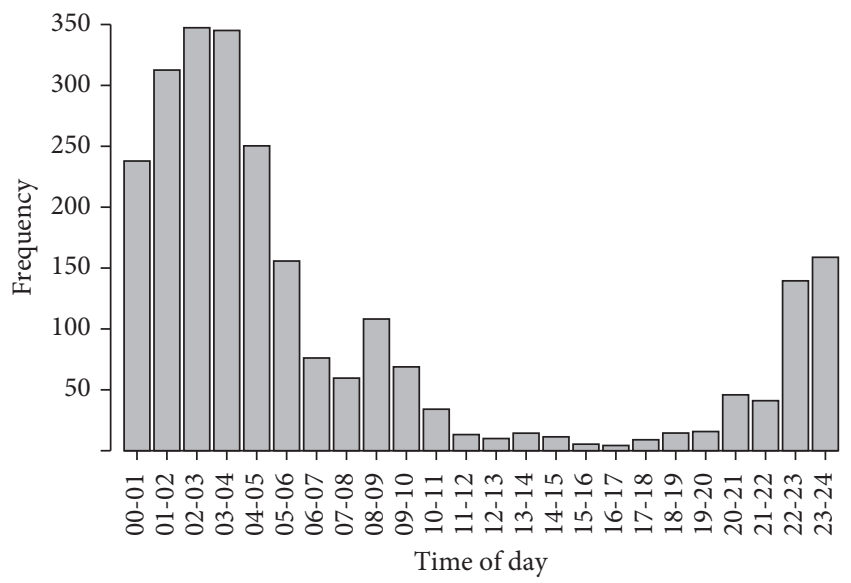

(b)

FIGURE 7: Temporal distribution of each quadrant: (a) quadrant I; (b) quadrant IV.

4.5. Analysis of the Spatial Characteristics. Next, the spatial characteristics were analyzed based on the availability belonging to the good and bad groups to investigate whether there were any imbalances in accessibility by region. The analysis showed that most administrative districts had good or poor availability at least one hour out of 24 hours a day because the level of availability changed according to the time of day. As a result, it was difficult to analyze the spatial characteristics because almost all regions belonged to good or bad groups if the availability of good and bad groups is expressed in terms of space.

Therefore, in this study, we analyzed the availability of the administrative districts in the top $15 \%$ based on the frequency of the administrative districts belonging to the good and bad groups. We did so in order to clearly understand the spatial distribution and characteristics of areas with good and poor accessibility. As a result, there were 35 administrative districts in the top 15\%, which had excellent accessibility, and they were spatially distributed in the outskirts of Seoul (as shown in Figure 8(a)). However, in the top $15 \%$, there were 50 administrative districts with very poor accessibility, as shown in Figure 8(b), and they were distributed mainly in the central areas of Seoul.

Such a spatial distribution might be caused by the following three reasons. First, most of the areas with good accessibility have DRT vehicle depots. Good accessibility 


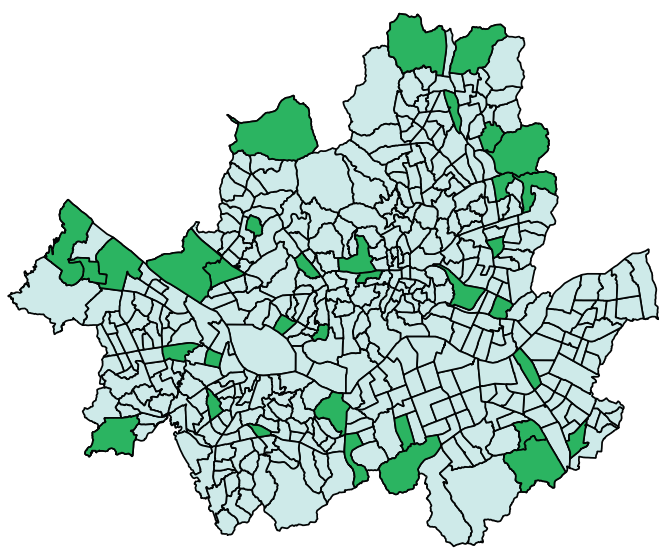

(a)

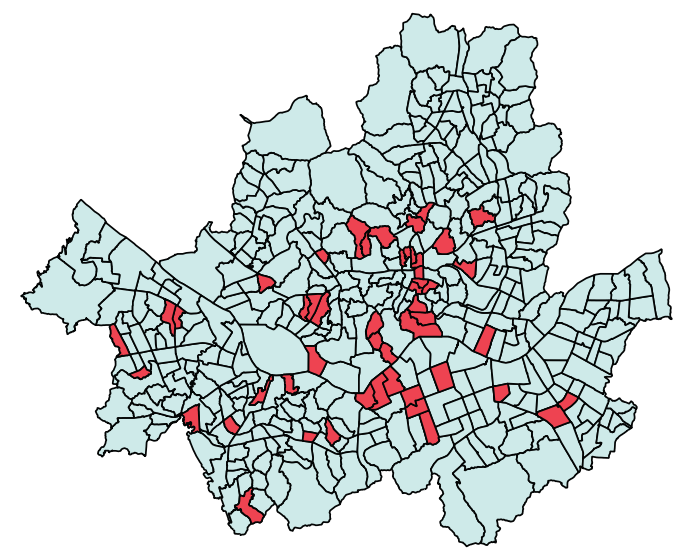

(b)

FIgURE 8: Spatial distribution of areas with good or poor accessibility: (a) top 15\% areas with good accessibility; (b) top 15\% areas with poor accessibility.

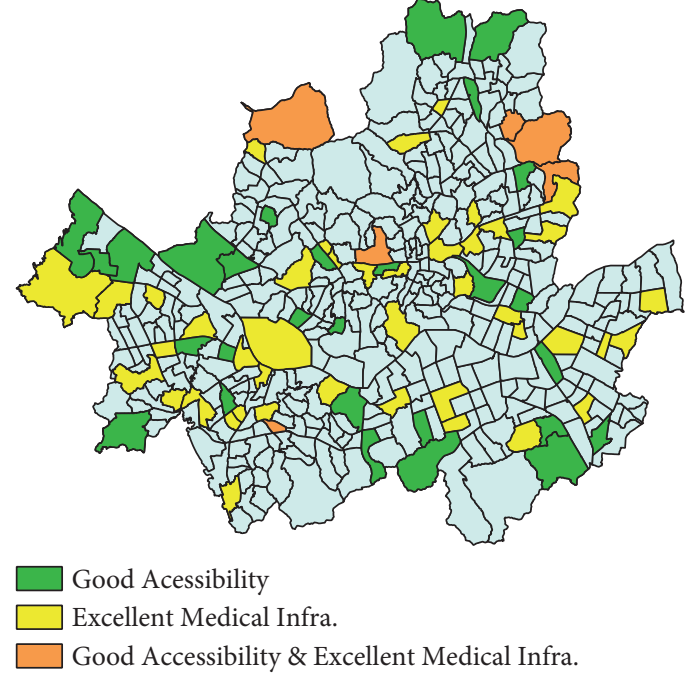

(a)

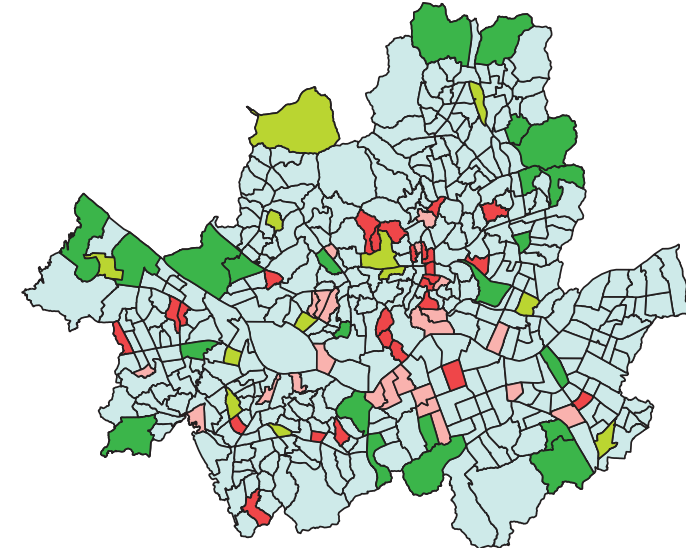

Good Accessibility \& Higher than average speed Good Accessibility \& Lower than average speed Poor Accessibility \& Higher than average speed Poor Accessibility \& Lower than average speed

(b)

FiguRE 9: Distribution of administrative districts with specific spatial properties: (a) distribution of administrative districts with excellent medical infrastructure and those with good accessibility; (b) classification of average speed standards in areas with good and poor accessibility.

means that there are many vehicles available in the area and that these vehicles stay in the area for a long time. Areas that have depots or are adjacent to depots are more likely to have available vehicles than other areas because vehicles far away from the depot take more time to return to the depot, so drivers prefer not to move far away from their depots. Another reason is that these depots have facilities where drivers can rest, and drivers tend to wait for the next dispatch at a depot close to where they are. The close relationship between these two variables is also explained through a correlation test $(\rho=0.2463, p-$ value $=0.000)$. Therefore, it is necessary to reallocate and expand depots to address this imbalance.

The second reason that causes this imbalance is the distribution of facilities that attract people with disabilities.
As a result of examining the purposes of trips of the DRT service for the disabled in 2019, about $70 \%$ of the trips were for medical services, including treatment, rehabilitation, and returning home. By considering these results, areas with high levels of medical infrastructure or healthcare services are expected to have a relatively greater influence on attracting users than other areas. Thus, areas that have high levels of medical infrastructure or are close to such areas are expected to have better accessibility than other areas. The fact that this relationship is valid might also be proved with a correlation coefficient $(\rho=0.0349, p-$ value $=0.0004)$.

Figure 9(a) shows the distribution of administrative districts (yellow color) in Seoul where tertiary general hospitals and general hospitals are located and where administrative districts with good accessibility are located 
(green color). As shown in the figure, most of the areas with excellent accessibility have the better medical infrastructure or are adjacent to areas with excellent medical infrastructure. This is due to the increased inflow of traffic using DRT services into the area and the vehicles waiting for their next dispatch after completing their service.

Finally, the differences in transportation environment factors by region cause imbalanced accessibility. Most drivers tend to avoid driving in areas with heavy traffic jams or during heavy traffic hours, so the areas with severe traffic congestion will have relatively poor accessibility because drivers are more likely to avoid them.

The average traffic speed data for each region were analyzed to investigate this phenomenon. The average traffic speed in Seoul is calculated using the speed data provided by the Korea transport data base (KTDB) was about $32.7 \mathrm{~km} / \mathrm{h}$. But among the 35 administrative districts in the top $15 \%$ with excellent accessibility, 23 administrative districts (65\%) were higher than the average traffic speed in Seoul. On the contrary, about $54 \%$ of the 50 administrative districts with very poor accessibility were lower than the average traffic speed in Seoul (Figure 9(b)). These results show that traffic congestion and accessibility are closely related and that traffic congestion is one of the factors that cause regional accessibility imbalances. The correlation coefficient also indicates that traffic congestion has a statistically significant relationship with accessibility $(\rho=0.2359, p-$ value $=0.000)$. Therefore, it is necessary to improve the traffic environment by investing in road infrastructure to resolve these imbalances.

\section{Conclusions}

Although everyone should be guaranteed the right to mobility, road user groups with reduced mobility due to disabilities or old age more often have their mobility rights infringed on than the normal adults. Therefore, many countries provide special means of transportation for mobility disadvantaged persons.

The Seoul metropolitan government also provides a DRT service for the disabled. This service operates according to the needs of passengers rather than on fixed routes or timetables, so the accessibility to use the service is not guaranteed equally in time and space, resulting in imbalances in the distribution of access in specific areas and periods.

This study attempted to identify the spatial and temporal imbalance of the DRT service for the disabled and suggested ways to improve the accessibility to DRT service. For this purpose, this study calculated the availability, an index that measures how easily and quickly call taxi services for the disabled are available at a specific time and area, and it defined and analyzed this index as a surrogate measure of accessibility to transportation.

The main findings are as follows. First, the accessibility to DRT service was not even over time. Service accessibility was relatively good during the daytime when many people are active, but the accessibility was poor during the late-night and early-morning hours. This phenomenon was caused by characteristics such as the change in supply according to time and drivers' breaks. Solving these problems by adjusting the drivers' service schedules will provide a more balanced DRT service in terms of time.

Second, the accessibility to DRT service had spatial characteristics that differed by region. The accessibility was excellent in areas that have depots or are adjacent to depots because these depots served as a base for the DRT service. Therefore, most of the spatial imbalance of accessibility can be resolved by increasing the number of depots, expanding them spatially, or reallocating them in administrative districts.

There also were administrative districts that had excellent medical infrastructure or that were near areas with good accessibility because the majority of DRT service calls were for the provision of medical services. This study only determined the level of medical infrastructure based on the location of large hospitals, but it could have analyzed the accessibility according to the spatial characteristics of the demand by the disabled in more detail by considering the facilities that are used frequently by call taxi users, such as rehabilitation facilities.

Finally, the imbalance in regional accessibility occurred due to differences in transportation environment factors by region. As a result of analyzing the traffic speed data by region, areas with high traffic speed had good accessibility, and the areas with heavy traffic jams had relatively poor accessibility. In reality, there are limits to explaining the spatial imbalance of accessibility with only one factor (traffic speed) because many different factors indicate the characteristics of regional traffic environments. Therefore, conducting additional research by considering various factors will yield implications for more realistic alternatives.

As mentioned above, the accessibility to transportation was tried to be explained by vehicle availability in this study, indicating that the accessibility might have an association with travel demands, but the relationship between the accessibility of the disabled and travel demands was not fully investigated because it was difficult to directly observe the potential travel demand. To address this phenomenon more realistically, a further study needs to be conducted in future with considering the characteristics of travel demand.

\section{Data Availability}

The data used to support the findings of this study are available from the corresponding author upon request.

\section{Conflicts of Interest}

The authors declare that there are no conflicts of interest regarding the publication of this paper.

\section{Acknowledgments}

This work was supported by the Basic Study and Interdisciplinary R\&D Foundation Fund of the University of Seoul (2021). The authors wish to express warm gratitude for the support. 


\section{References}

[1] U. N. G. Assembly, "Universal declaration of human rights," UN General Assembly, vol. 302, no. 2, pp. 14-25, 1948.

[2] S. H. K. Soltani, M. Sham, M. Awang, and R. Yaman, "Accessibility for disabled in public transportation terminal," Procedia - Social and Behavioral Sciences, vol. 35, pp. 89-96, 2012.

[3] I. Casas, "Social exclusion and the disabled: an accessibility Approach*," The Professional Geographer, vol. 59, no. 4, pp. 463-477, 2007.

[4] P. Bakker and J. Hal, "Understanding Travel Behaviour of People with a travel-impeding handicap: each trip counts," in Proceedings of the 11th International Conference on Mobility and Transport for Elderly and Disabled people, Montreal, Canada, June 2007.

[5] V. Canal-Fernandez and M. N. Muniz, "An exploratory analysis of disabled people accessibility to urban public transport: the use of Geographical Information Systems," Investigaciones Reginales, vol. 30, pp. 79-101, 2014.

[6] L. Ferrari, M. Berlingerio, F. Calabrese, and J. Reades, "Improving the accessibility of urban transportation networks for people with disabilities," Transportation Research Part C: Emerging Technologies, vol. 45, pp. 27-40, 2014.

[7] C. E. Kirchner, E. G. Gerber, and B. C. Smith, "Designed to deter," American Journal of Preventive Medicine, vol. 34, no. 4, pp. 349-352, 2008.

[8] A. K. M. Abir and M. S. Hoque, "As study on mobility problem of disabled people in Dhaka city," 4th Annual Paper Meet and 1st Civil Engineering Congress, pp. 152-161, Dhaka Bangladesh University of Engineering and Technology, Dhaka Bangladesh, 2011.

[9] T. Henry, "Policy on persons with disabilities," Office of the Prime Minister, (social Services Delivery), 2009.

[10] S. W. Chung and J. H. Rho, "Case study of barrier free design for transportation vulnerable: focusing on transfer station complex in Seoul station," Journal of the Korea AcademiaIndurstrial cooperation Society, vol. 18, no. 3, pp. 333-344, 2017.

[11] L. Yin Mun, W. Kun Xin, and S. Devi Rajendran, "A study on the barriers in accessibility for the disabled at bus terminal," E3S Web of Conferences, vol. 136, Article ID 04084, 2019.

[12] Y. J. Wu, W. J. Liu, and C. H. Yuan, “A mobile-baded barrierfree service transportation platform for people with disabilities," Computers in Human Behavior, vol. 107, Article ID 105776, 2020.

[13] J. L. Bezyak, S. A. Sabella, and R. H. Gattis, "Public transportation: an investigation of barriers for people with disabilities," Journal of Disability Policy Studies, vol. 28, no. 1, pp. 52-60, 2017.

[14] C. Bhat, S. Handy, K. Kockelman et al., "Development of an urban accessibility index: formulations, aggregation, and application," Center for Transportation Research The University of Texas at Austin, pp. 4938-4944, 2002.

[15] A. Golub and K. Martens, "Using principles of justice to assess the modal equity of regional transportation plans," Journal of Transport Geography, vol. 41, pp. 10-20, 2014.

[16] R. H. M. Pereira, D. Banister, T. Schwanen, and N. Wessel, "Distributional effects of transport policies on inequalities in access to opportunities in Rio de Janeiro," Journal of Transport and Land Use, vol. 12, no. 1, pp. 741-764, 2019.

[17] Y. Fan, A. Guthrie, and D. Levinson, "Impact of light-rail implementation on labor market accessibility: a transportation equity perspective," The Journal of Transport and Land Use, vol. 5, no. 3, pp. 28-39 2012.

[18] S. Feng, L. Chen, R. Sun et al., "The distribution and accessibility of urban parks in beijing, China: implications of social equity," International Journal of Environmental Research and Public Health, vol. 16, no. 24, Article ID 4894, 2019.

[19] T. F. Welch and S. Mishra, "A measure of equity for public transit connectivity," Journal of Transport Geography, vol. 33, pp. 29-41, 2013.

[20] R. Liu, Y. Chen, J. Wu, T. Xu, L. Gao, and X. Zhao, "Mapping spatial accessibility of public transportation network in an urban area - a case study of Shanghai Hongqiao Transportation Hub," Transportation Research Part D: Transport and Environment, vol. 59, pp. 478-495, 2018.

[21] T. Zuo, H. Wei, N. Chen, and Z. Chun, "First-and-last mile via bicycling to improving transit accessibility and advancing transportation equity," Cities, vol. 99, Article ID 102614, 2020.

[22] Y. Chen, S. Ravulaparthy, K. Dalal et al., "Development of indicators of opportunity-based accessibility," Transportation Research Record: Journal of the Transportation Research Board, vol. 2255, no. 1, pp. 58-68, 2011. 